\title{
Non prompt D-meson measurements with ALICE at the LHC
}

\author{
Marianna Mazzilli on behalf of the ALICE Collaboration ${ }^{1, \star}$ \\ ${ }^{1}$ Dipartimento Interateneo di Fisica 'M.Merlin' and Sezione INFN, Bari, Italy
}

\begin{abstract}
The production of hadrons with open heavy flavour (charm and beauty) in high-energy nucleus-nucleus collisions is a powerful tool to study the properties of the deconfined phase of strongly interacting matter known as the Quark-Gluon Plasma (QGP). The production of charm and beauty quarks occurs in hard partonic scattering processes in the early stage of the collisions. ALICE is the LHC experiment devoted to the study of heavy-ion physics. It is able to reconstruct charmed mesons in exclusive decays (e.g. $\mathrm{D}^{0} \rightarrow \mathrm{K}^{-} \pi^{+}$) and beauty hadrons in semi-inclusive decays (e.g. $\mathrm{B} \rightarrow \mathrm{eX}, \mathrm{B} \rightarrow \mathrm{J} / \psi \mathrm{X}$ ). At LHC energies a significant component of the inclusive D-meson yield originates from the decay of beauty-flavoured hadrons, whose knowledge is essential to determine the production of prompt $\mathrm{D}$ mesons coming from charm quarks. A precise determination of the non-prompt fraction combined with the determination of the inclusive D-meson yield would allow a measurement of beauty production. A data-driven method that exploits the different shapes of the distributions of the transverse-plane impact parameter to the primary vertex of prompt and feed-down $\mathrm{D}$ mesons in $\mathrm{p}-\mathrm{Pb}$ collisions is used in ALICE. An alternative approach based on the $\mathrm{D}$-meson decay length for $\mathrm{Pb}-\mathrm{Pb}$ collisions is under study.
\end{abstract}

\section{Introduction}

The ALICE experiment at the LHC investigates the properties of the Quark-Gluon Plasma, the hot and dense state of strongly-interacting matter produced in high-energy heavy-ion collisions. Charm and beauty quarks are among the most powerful probes to study the QGP in heavy-ion collisions: they are produced in hard parton scattering processes occurring in the early stages of the collision, traverse the QCD medium and interact with its constituents experiencing the whole evolution of the medium. Charm production in $\mathrm{pp}, \mathrm{p}-\mathrm{Pb}$ and $\mathrm{Pb}-\mathrm{Pb}$ collisions is studied in ALICE, e.g. by reconstructing exclusive D-meson hadronic decays. The yield obtained from the fit to the invariant mass distribution of D-meson candidates includes contributions from both prompt D mesons (i.e. produced at the primary interaction vertex from the charm quark, either directly or through decays of excited open charm and charmonium states) and feed-down (i.e. originating from beauty-hadron decays). The fraction of prompt $\mathrm{D}$ mesons in the raw yield, $f_{\text {prompt }}$, can be extracted using different approaches. The first method employs perturbative QCD (pQCD) calculations, namely FONLL [1][2], in order to obtain the fraction of prompt and feed-down D-meson cross sections (FONLL-based method). A datadriven approach for the extraction of $f_{\text {prompt }}$ has also been used in ALICE [3]. The method exploits the different shapes of the distributions of the transverse-plane impact parameter to the primary vertex

\footnotetext{
^e-mail: marianna.mazzilli@ba.infn.it
} 
$\left(d_{0}\right)$ of prompt and feed-down D mesons. An alternative approach based on the pseudo-proper decay length is under study.

\section{D-meson reconstruction}

$\mathrm{D}^{0}, \mathrm{D}^{+}, \mathrm{D}^{*+}$ and $\mathrm{D}_{\mathrm{s}}^{+}$mesons, and their charge conjugates, are reconstructed via their hadronic decay channels $\mathrm{D}^{0} \rightarrow \mathrm{K}^{-} \pi^{+}$(with a branching ratio, $\mathrm{BR}$, of $3.88 \pm 0.05 \%$ ), $\mathrm{D}^{+} \rightarrow \mathrm{K}^{+} \pi^{-} \pi^{+}$ $(\mathrm{BR}=9.13 \pm 0.19 \%), \mathrm{D}^{*+} \rightarrow \mathrm{D}^{0} \pi^{+}(\mathrm{BR}=67.7 \pm 0.5 \%)$ followed by $\mathrm{D}^{0} \rightarrow \mathrm{K}^{-} \pi^{+}$, and $\mathrm{D}_{\mathrm{s}}^{+} \rightarrow \phi \pi^{+} \rightarrow \mathrm{K}^{-} \mathrm{K}^{+} \pi^{+}(\mathrm{BR}=2.24 \pm 0.1 \%)$ [4]. The $\mathrm{D}^{0}, \mathrm{D}^{+}$, and $\mathrm{D}_{\mathrm{s}}^{+}$mesons decay weakly with mean proper decay lengths (c $\tau$ ) of about 123,312 and $150 \mu \mathrm{m}$ [4], respectively. The analysis strategy is based on the reconstruction of secondary vertices separated by a few hundred $\mu \mathrm{m}$ from the interaction point. Both primary and secondary vertices are reconstructed with the Inner Tracking System (ITS) [7]. The selection of the D-meson decay topology is mainly based on the displacement of the tracks from the interaction vertex, the separation of the primary and secondary vertices, and the pointing of the reconstructed D-meson momentum to the primary vertex [5,6]. Further reduction of the combinatorial background is obtained by applying particle identification (PID) with the Time Projection Chamber (TPC) and the Time Of Flight detector (TOF) for pion and kaon tracks [7]. The applied PID strategy provides a reduction of the combinatorial background by a factor of about three at low transverse momentum $\left(p_{\mathrm{T}}\right)$ while preserving an efficiency of $95 \%$ for the $\mathrm{D}^{0}, \mathrm{D}^{+}$and $\mathrm{D}^{*+}$ signals and of $85 \%$ for the $\mathrm{D}_{\mathrm{s}}^{+}$signal. The D-meson raw yields are extracted from fits to the $\mathrm{D}^{0}, \mathrm{D}^{+}$ and $\mathrm{D}_{\mathrm{s}}^{+}$candidate invariant-mass distributions and to the mass difference $\Delta M=M(\mathrm{~K} \pi \pi)-M(\mathrm{~K} \pi)$ distributions for $\mathrm{D}^{*+}$ candidates. In the fit function, the signal is modeled with a Gaussian and the background is described by an exponential term for $\mathrm{D}^{0}, \mathrm{D}^{+}$and $\mathrm{D}_{\mathrm{s}}^{+}$candidates and by a threshold function multiplied by an exponential for the $\mathrm{D}^{*+}$ case. For all four $\mathrm{D}$-meson species, the mean values of the Gaussian functions in all transverse momentum and rapidity intervals were found to be compatible within uncertainties with the PDG world average values [4]. Figure 1 shows the $\mathrm{D}^{0}$ and $\mathrm{D}^{+}$ candidate invariant mass and $\mathrm{D}^{*+}$ mass-difference distribution in $\mathrm{Pb}-\mathrm{Pb}$ collisions in three $p_{\mathrm{T}}$ intervals for the centrality ${ }^{1}$ class $0-10 \%$ [5].

The D-meson raw yields extracted in each $p_{\mathrm{T}}$ and $y$ interval include contributions from both prompt and from feed-down D mesons. The raw yields were corrected by a factor $\left(f_{\text {prompt }}\right)$ in order to obtain the prompt D-meson cross sections:

$$
\frac{\mathrm{d}^{2} \sigma^{\mathrm{D}}}{\mathrm{d} p_{\mathrm{T}} d y}=\frac{1}{2} \frac{1}{\Delta p_{\mathrm{T}}} \cdot \frac{f_{\text {prompt }} \cdot N^{\mathrm{D}+\overline{\mathrm{D}}, \mathrm{raw}}\left(p_{\mathrm{T}}, y\right)}{\Delta y} \cdot \frac{1}{(\operatorname{Acc} \times \epsilon)_{\mathrm{prompt}}\left(p_{\mathrm{T}}\right)} \cdot \frac{1}{\mathrm{BR} \cdot L_{\mathrm{int}}}
$$

In the formula, $N^{\mathrm{D}+\overline{\mathrm{D}} \text {,raw }}$ is the raw yield (sum of particles and antiparticles). The factor $1 / 2$ accounts for the fact that the measured yields include particles and antiparticles while the cross sections are given for particles only; $f_{\text {prompt }}$ is the fraction of prompt $\mathrm{D}$ mesons in the raw yield; (Acc $\left.\times \epsilon\right)_{\text {prompt }}$ is the product of acceptance and efficiency for prompt D-mesons, where $\epsilon$ accounts for the D-meson decay track reconstruction and selection and for D-meson candidate selection with secondary vertex and PID cuts; $\Delta p_{\mathrm{T}}$ and $\Delta y$ are the widths of the transverse momentum and rapidity intervals; $\mathrm{BR}$ is the branching ratio of the considered decay channel, and $L_{\text {int }}$ is the integrated luminosity. The acceptance and efficiency correction factors were obtained from Monte Carlo simulations including detailed descriptions of the geometry of the apparatus and of the detector response.

\footnotetext{
${ }^{1}$ Centrality is a variable that describes the geometry of a $\mathrm{Pb}-\mathrm{Pb}$ collision in terms of percentiles of the total hadronic $\mathrm{Pb}-\mathrm{Pb}$ cross section. Lowest percentiles correspond to lowest values of the impact parameter between the two colliding nuclei.
} 

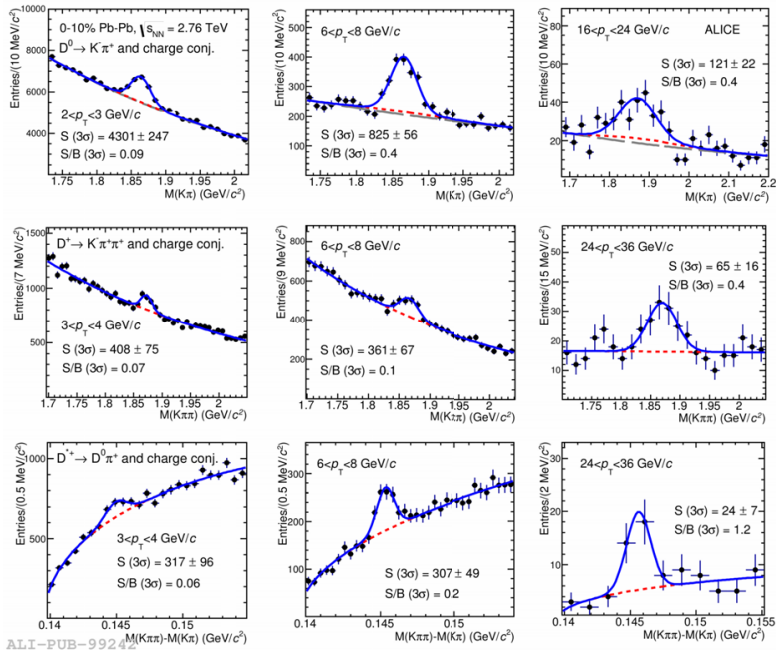

Figure 1. Invariant mass distributions for $\mathrm{D}^{0}$ (top row) and $\mathrm{D}^{+}$(central row) candidates, and mass difference distribution for $\mathrm{D}^{*+}$ candidates $\Delta M=M(\mathrm{~K} \pi \pi)-M(\mathrm{~K} \pi)$ (bottom row) for the centrality class $0-10 \%$ in $\mathrm{Pb}-\mathrm{Pb}$ collisions at $\sqrt{s_{\mathrm{NN}}}=2.76 \mathrm{TeV}$. The distributions are reported in three $p_{\mathrm{T}}$ intervals for each meson (left, middle and right column).

\section{Prompt D-meson fraction}

\subsection{FONLL-based method}

The FONLL-based method estimates $f_{\text {prompt }}$ as:

$f_{\text {prompt }}=1-\frac{N^{\mathrm{D}+\overline{\mathrm{D}}_{\text {,feed-down }}}}{N^{\mathrm{D}+\overline{\mathrm{D}}_{\text {,raw }}}}=1-R_{\mathrm{AA}}^{\mathrm{feed-down}} \cdot\left\langle T_{\mathrm{AA}}\right\rangle\left(\frac{\mathrm{d} \sigma}{\mathrm{d} p_{\mathrm{T}}}\right)_{\text {feed-down },|y|<0.5}^{\mathrm{FONLL}} \frac{\Delta p_{\mathrm{T}} \Delta y(\operatorname{Acc} \times \epsilon)_{\text {feed-down }} \cdot \mathrm{BR} \cdot L_{\mathrm{int}}}{N_{\text {raw }}^{\mathrm{D}+\overline{\mathrm{D}} / 2}}$

The procedure uses the B-meson production cross section in pp collisions estimated with FONLL calculations $[1,6,8,10]$, the $\mathrm{B} \rightarrow \mathrm{D}+\mathrm{X}$ decay kinematics from the EvtGen package [9] and the efficiencies for D-meson reconstruction from beauty-hadron decays obtained from Monte Carlo simulations. Different hypotheses on the nuclear modification factor $R_{\mathrm{AA}}^{\text {feed-down }}$, defined as the ratio of the $p_{\mathrm{T}}$-differential yield of $\mathrm{D}$ mesons from $\mathrm{B}$ decays in $\mathrm{Pb}-\mathrm{Pb}$ collisions to the corresponding cross section in pp collisions scaled by the average nuclear overlap function $\left(\left\langle T_{\mathrm{AA}}\right\rangle\right)$, are also introduced. The resulting $f_{\text {prompt }}$ values and their uncertainties are shown in the right-hand panels of Fig.2 for $\mathrm{D}^{0}, \mathrm{D}^{+}$and $\mathrm{D}^{*+}$ mesons in the $\left|y_{\text {lab }}\right|<y_{\text {fid }}\left(p_{\mathrm{T}}\right)$ interval $\left(y_{\mathrm{fid}} \sim 0.5-0.8\right.$ depending on the $\left.p_{\mathrm{T}}\right)$. The central values of $f_{\text {prompt }}$ range between 0.81 and 0.96 depending on D-meson species and $p_{\mathrm{T}}$ with no significant rapidity dependence.

\subsection{Data-driven method}

The data-driven method exploits the different shapes of the distributions of the transverse-plane impact parameter to the primary vertex $\left(d_{0}\right)$ of prompt and feed-down D mesons and estimates the prompt fraction $\left(f_{\text {prompt }}\right)$ via an unbinned likelihood fit of the $d_{0}$ distribution of $\mathrm{D}^{0}\left(\mathrm{D}^{+}\right)$-meson candidates 

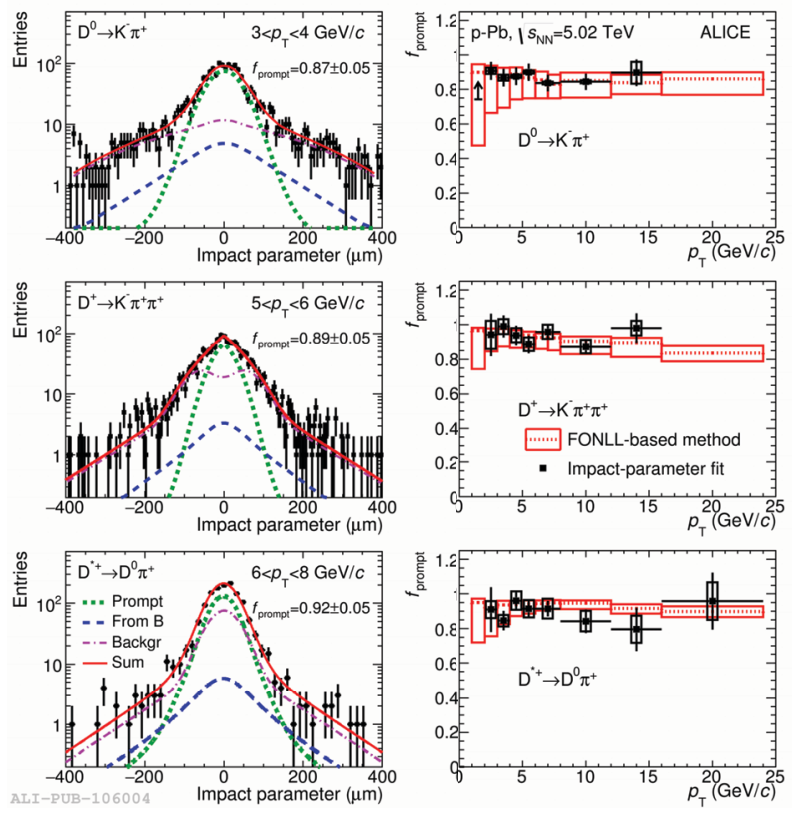

Figure 2. Left: Examples of fits to $\mathrm{D}^{0}$ (top), $\mathrm{D}^{+}$(middle) and $\mathrm{D}^{*+}$ (bottom) impact-parameter distributions for p - Pb collisions at $\sqrt{s_{\mathrm{NN}}}=5.02 \mathrm{TeV}$ in three $p_{\mathrm{T}}$ intervals: $3<p_{\mathrm{T}}<4 \mathrm{GeV} / c, 5<p_{\mathrm{T}}<6 \mathrm{GeV} / c$ and $6<p_{\mathrm{T}}<$ $8 \mathrm{GeV} / c$. The curves show the fit functions describing the prompt, feed-down and background contributions, as well as their sum. Right: fraction of prompt $\mathrm{D}^{0}$ (top), $\mathrm{D}^{+}$(middle) and $\mathrm{D}^{*+}$ (bottom) raw yield as a function of $p_{\mathrm{T}}$ compared to the FONLL-based approach (red lines). The results from the data-driven method are shown as square markers with the error bars (boxes) representing the statistical (systematic) uncertainty. The arrow in the interval $1<p_{\mathrm{T}}<2 \mathrm{GeV} / c$ represents the minimum value within a $95 \%$ confidence level. The central values of $f_{\text {prompt }}$ from the FONLL-based approach are shown by the dashed line and their uncertainty by the red boxes.

with invariant mass $\left|\mathrm{M}-\mathrm{M}_{\mathrm{D}}\right|<1.5(2) \sigma$ (where $\sigma$ is the width of the Gaussian function describing the $\mathrm{D}$-meson signal in the invariant-mass fits) and of $\mathrm{D}^{*+}$-meson candidates with a mass difference $\left|\Delta \mathrm{M}-\Delta \mathrm{M}_{D^{*+}}\right|<2.5 \sigma$. The fit function used is [3]:

$$
F\left(d_{0}\right)=S \cdot\left[\left(1-f_{\text {prompt }}\right) F^{\text {feed-down }}\left(d_{0}\right)+f_{\text {prompt }} F^{\text {prompt }}\left(d_{0}\right)\right]+B \cdot F^{\text {backgr }}\left(d_{0}\right)
$$

In this function, $\mathrm{S}$ and $\mathrm{B}$ are, respectively, the signal and background raw yields in the selected invariant-mass range; $F^{\text {prompt }}\left(d_{0}\right), F^{\text {feed-down }}\left(d_{0}\right)$ and $F^{\text {backgr }}\left(d_{0}\right)$ are functions describing the impact parameter distributions of prompt D mesons, feed-down D mesons, and background, respectively. The function $F^{\text {prompt }}$ is a detector resolution term and is modeled with a Gaussian and a symmetric exponential term, describing the tails of the impact-parameter distribution of prompt $\mathrm{D}$ mesons. $F^{\text {feed-down }}$ is the convolution of the detector resolution term with a symmetric double-exponential function $\left(F_{\text {true }}^{\text {feed-down }}\right)$ describing the intrinsic impact parameter distribution of secondary D mesons from B-meson decays, which is determined by the decay length and decay kinematics of B mesons. $F^{\text {backgr }}$ is the sum of a Gaussian and a symmetric exponential term centered at zero. For $\mathrm{D}^{+}$mesons, the background impact-parameter distribution has a double-peak structure with a depletion around zero induced by the selections applied. The shape is thus modeled with two Gaussians and two symmetric exponential terms. The parameters of $F^{\text {backgr }}$ are fixed by fitting the impact parameter 
distribution of background candidates in the side-bands of the signal peak in the invariant-mass distributions. Figure 2 (left) shows examples of fits to the impact-parameter distributions of $\mathrm{D}^{0}, \mathrm{D}^{+}$and $\mathrm{D}^{*+}$ mesons in the transverse-momentum intervals $3<p_{\mathrm{T}}<4 \mathrm{GeV} / c, 5<p_{\mathrm{T}}<6 \mathrm{GeV} / c$ and $6<p_{\mathrm{T}}<$ $8 \mathrm{GeV} / c$, respectively. The prompt fraction of $\mathrm{D}^{0}, \mathrm{D}^{+}$and $\mathrm{D}^{*+}$ mesons measured with this method is shown in Fig. 2 (right). For the interval $1<p_{\mathrm{T}}<2 \mathrm{GeV} / c$, given the limited precision of the impactparameter fit, a lower limit could be estimated only for $\mathrm{D}^{0}$ mesons at a $95 \%$ confidence level on the basis of the statistical and systematic uncertainties. For the same reason, in the highest $p_{\mathrm{T}}$ interval, $16<p_{\mathrm{T}}<24 \mathrm{GeV} / c$, the prompt fraction could be determined with the data-driven method only for $\mathrm{D}^{*+}$ mesons. The prompt fraction measured with the impact-parameter fits is found to be compatible with the FONLL-based estimation within uncertainties. For $\mathrm{D}^{0}$ mesons, the data-driven approach provides a more precise determination of the prompt fraction [3], while for $\mathrm{D}^{*+}$ and $\mathrm{D}^{+}$mesons smaller uncertainties are obtained with the FONLL-based method.

\subsection{Method based on pseudo-proper decay length}

A new method is under study for the separation of the prompt D-meson fraction from the non-prompt component in $\mathrm{Pb}-\mathrm{Pb}$ collisions. This new approach is based on a fit to the distribution of the pseudoproper decay length variable, $x$, which is obtained by considering the scalar variable $L_{x y}$, defined as the signed projection of the $\mathrm{D}^{0}$ flight distance onto its transverse momentum vector:

$$
L_{x y}=\vec{L} \cdot \frac{\vec{p}_{\mathrm{T}}^{D}}{\left|\vec{p}_{\mathrm{T}}^{\mathrm{D}}\right|}
$$

The pseudo proper decay length is then defined as:

$$
x=\frac{L_{x y} \cdot M^{\mathrm{D}} \cdot c}{\left|\vec{p}_{\mathrm{T}}^{\mathrm{D}}\right|}
$$

Here $\vec{L}$ is the vector pointing from the primary vertex to the $\mathrm{D}^{0}$ decay vertex and $M^{\mathrm{D}}$ the mass of the $\mathrm{D}^{0}$ taken from [4]. This approach would achieve the separation of the two components exploiting the different shapes of the pseudo-proper decay distributions of prompt and feed-down D mesons. The pseudo-proper decay method was successfully employed for the measurement of prompt and non-prompt $\mathrm{J} / \psi$ in $\mathrm{pp}[11]$ and $\mathrm{Pb}-\mathrm{Pb}$ collisions [12].

\section{References}

[1] M. Cacciari, S. Frixione, N. Houdeau, M. L. Mangano, P. Nason, G. Ridolfi, JHEP 10 (2012) 137.

[2] M. Cacciari, M. Greco, and P. Nason, JHEP 05 (1998) 007.

[3] J. Adam et al. [ALICE Collaboration], CERN-EP-2016-127, arXiv:1605.07569.

[4] K. A. Olive et al. [Particle Data Group Collaboration], Chin. Phys. C38 (2014) 090001.

[5] J. Adam et al. [ALICE Collaboration], JHEP 03 (2016) 081.

[6] B. Abelev et al. [ALICE Collaboration], JHEP 01 (2012) 128.

[7] B. Abelev et al. [ALICE Collaboration], Int. J. Mod. Phys. A29 (2014) 1430044.

[8] B. Abelev et al. [ALICE Collaboration], JHEP 07 (2012) 191.

[9] D. J. Lange, Nucl. Inst. and Meth. in Phys. Res. Section A 462 no. 1 - 2, (2001) 152 - 155.

[10] LHCb Collaboration, R. Aaij et al., Nucl. Phys. B871 (2013) 1-20.

[11] B. Abelev et al. [ALICE Collaboration], JHEP 11 (2012) 065.

[12] J. Adam et al. [ALICE Collaboration], JHEP 07 (2015) 051. 